\title{
Whole genome sequencing of Trypanosoma cruzi field isolates reveals extensive genomic variability and complex aneuploidy patterns within Tcll DTU
}

João Luís Reis-Cunha', Rodrigo P. Baptista ${ }^{1,2}$, Gabriela F. Rodrigues-Luiz ${ }^{1,3}$, Anderson Coqueiro-dos-Santos', Hugo O. Valdivia 1,4, Laila Viana de Almeida', Mariana Santos Cardoso', Daniella Alchaar D'Ávila', Fernando Hugo Cunha Dias', Ricardo Toshio Fujiwara', Lúcia M. C. Galvão', Egler Chiari', Gustavo Coutinho Cerqueira ${ }^{5}$ and Daniella C. Bartholomeu ${ }^{1^{*}}$ (i)

\begin{abstract}
Background: Trypanosoma cruzi, the etiologic agent of Chagas disease, is currently divided into six discrete typing units (DTUs), named Tcl-TcVI. Tcll is among the major DTUs enrolled in human infections in South America southern cone, where it is associated with severe cardiac and digestive symptoms. Despite the importance of Tcll in Chagas disease epidemiology and pathology, so far, no genome-wide comparisons of the mitochondrial and nuclear genomes of Tcll field isolates have been performed to track the variability and evolution of this DTU in endemic regions.
\end{abstract}

Results: In the present work, we have sequenced and compared the whole nuclear and mitochondrial genomes of seven Tcll strains isolated from chagasic patients from the central and northeastern regions of Minas Gerais, Brazil, revealing an extensive genetic variability within this DTU. A comparison of the phylogeny based on the nuclear or mitochondrial genomes revealed that the majority of branches were shared by both sequences. The subtle divergences in the branches are probably consequence of mitochondrial introgression events between Tcll strains. Two T. cruzi strains isolated from patients living in the central region of Minas Gerais, S15 and S162a, were clustered in the nuclear and mitochondrial phylogeny analysis. These two strains were isolated from the other five by the Espinhaço Mountains, a geographic barrier that could have restricted the traffic of insect vectors during T. cruzi evolution in the Minas Gerais state. Finally, the presence of aneuploidies was evaluated, revealing that all seven Tcll strains have a different pattern of chromosomal duplication/loss.

Conclusions: Analysis of genomic variability and aneuploidies suggests that there is significant genomic variability within Minas Gerais Tcll strains, which could be exploited by the parasite to allow rapid selection of favorable phenotypes. Also, the aneuploidy patterns vary among T. cruzi strains and does not correlate with the nuclear phylogeny, suggesting that chromosomal duplication/loss are recent and frequent events in the parasite evolution.

Keywords: Trypanosoma cruzi, Tcll, Field isolates, Genomic variability, Copy number variation, Ploidy

\footnotetext{
* Correspondence: daniella@icb.ufmg.br

${ }^{1}$ Departamento de Parasitologia, Universidade Federal de Minas Gerais, Belo

Horizonte, Brazil

Full list of author information is available at the end of the article
}

(c) The Author(s). 2018 Open Access This article is distributed under the terms of the Creative Commons Attribution 4.0 International License (http://creativecommons.org/licenses/by/4.0/), which permits unrestricted use, distribution, and reproduction in any medium, provided you give appropriate credit to the original author(s) and the source, provide a link to the Creative Commons license, and indicate if changes were made. The Creative Commons Public Domain Dedication waiver (http://creativecommons.org/publicdomain/zero/1.0/) applies to the data made available in this article, unless otherwise stated. 


\section{Background}

The protozoan parasite Trypanosoma cruzi is the etiologic agent of Chagas disease, a chronic debilitating illness that is endemic in Latin America, affecting 5-8 million people and accounting for 662,000 disability adjusted life years [1-3]. Due to its extreme genomic and phenotypic variability, $T$. cruzi is currently divided into six discrete typing units (DTUs), named TcI - TcVI $[4,5]$. The inclusion of a new DTU, Tcbat, which comprises bat-restricted trypanosomes, is still under debate $[5,6]$. From the six DTUs, TcI, TcII, TcV and TcVI are usually involved with the domestic cycle of Chagas disease, accounting for the majority of the human infections [5]. Human infections caused by TcI strains are more prevalent from Central America to Bolivia, while TcII, TcV and TcVI human infections are more common in the Southern cone of South America, encompassing countries as Argentina, Chile, Paraguay, Bolivia and Brazil [5, 7-9]. Recently, several TcVI parasites were isolated from humans and vectors in Colombia, suggesting that the distribution of this DTU could be broader than previously speculated [10]. Although the division in six DTUs is well accepted, there are four major proposed models to explain T. cruzi evolutionary history [11-14]. Even though these models disagree about the ancestral strains and the number of hybridization events during T. cruzi evolution, they all agree that $\mathrm{TcV}$ and $\mathrm{TcVI}$ are hybrids, originated from parental TcII and TcIII strains. It is still unknown if these hybrids arose from a single hybridization [15] or from multiple independent recombination events [14, 16, 17]. Molecular dating suggests that these two hybrid lineages evolved recently; reinforcing the assumption that genetic exchange could still be driving the emergence of $T$. cruzi recombinant isolates $[15,16]$.

The prevalent hypothesis that $T$. cruzi replication is mostly clonal [18-20] is been confronted by several recent findings suggesting that recombination events are frequent in $T$. cruzi populations from close geographic regions [14, 21-24]. The majority of field evidences suggest that recombination in $T$. cruzi is a non-obligatory, but common feature, and that parasexual mechanisms could be involved in genetic exchange processes in this parasite $[16,17,21,25,26]$. In fact, the presence of Chromosomal Copy Number Variation (CCNV), the duplication or loss of whole chromosome sequences, in T. cruzi $[27,28]$ could be a result of a fusion of diploid parasite cells followed by genome erosion, in a similar way as the Candida albicans parasexual cycle [17, 29-31]. According to this model, during the mammalian stage of the infection, the nucleus of two parasite cells fuse, resulting in a polyploid progeny, which may lose some supernumerary chromosomes resulting in different degrees of chromosomal aneuploidies [17, 32]. This assumption is further supported by the subtetraploidy found in $T$. cruzi experimental hybrids [23, 30], and by the $\sim 70 \%$ higher DNA content in hybrids when compared to parental strains [33, 34]. Recent experiments based on tiling arrays [27], or whole genome sequencing [28], have showed that CCNV vary among and even within $T$. cruzi DTUs, suggesting that aneuploidy is a common feature in this parasite. However, the extent of chromosomal variation in close-related field isolates, or even the rate in which these chromosomal duplication/deletion events occur in $T$. cruzi is still unknown.

T. cruzi belongs to Kinetoplastida order, which is characterized by having the mitochondrial genome composed by $\sim 30$ copies of 20-50 kb maxicircles and thousands of copies of $\sim 1 \mathrm{~kb}$ minicircles, which together comprise the kinetoplast DNA (kDNA) [26, 35]. Maxicircles are the functional equivalent of eukaryotic mitochondrial DNA, encoding genes that in these parasites can be edited by the RNA editing machinery that lead to U-insertions/deletions directed by minicircle sequences to correct frameshifts and premature stop codons [36-39]. Minicircle sequences are highly heterogeneous even within a single clone [40], while maxicircle sequences are relatively homogeneous, at least within their coding regions which represent $\sim 63 \%$ of the T. cruzi maxicircle genome [26]. Phylogenetic analyses of $T$. cruzi maxicircles lead to the identification of three mitochondrial clades: clade A comprising TcI maxicircles; clade B comprising TcIII, TcIV, TcV and TcVI maxicircles; and clade $\mathrm{C}$ comprising TcII maxicircles $[11,26]$. Although maxicircle sequences are usually conserved within a DTU, several studies have showed evidences of intra-lineage mitochondrial introgression, where the maxicircle genome from a DTU is associated with a non-recombinant nuclear genome of a different DTU $[11,17,25,26,41]$. Besides introgression, the occurrence of minor heteroplasmy, a presence of heterogeneous mitochondrial genomes in an individual cell, has also been reported in the T. cruzi Sylvio X10 strain $[17,26]$. The mechanism behind both processes as well as their importance to T. cruzi evolution is still unknown, however these processes could be important to satisfy the necessity to escape Muller's ratchet, the irreversible accumulation of deleterious mutations resulting from clonal reproduction $[17,23]$.

The majority of TcII infections occur in South American countries, such as Brazil and Argentina, being responsible for severe acute infections and by chronical mixed symptomatology with megaesophagus/megacolon and chagasic cardiomegaly [5, 11, 42]. Despite the importance of TcII in Chagas disease epidemiology and pathology, so far, no genome-wide comparisons of TcII field isolates have been performed to track the variability and evolution of this DTU in endemic regions. In the present work, we have sequenced the whole nuclear and mitochondrial genomes of seven TcII strains, which were 
recently isolated from chagasic patients with the indeterminate or cardiac forms of Chagas disease. These isolates where originated from the central and northeastern regions of Minas Gerais state, Brazil, an endemic region for T. cruzi TcII infection. We evaluated and compared the phylogeny of these TcII field isolates, as well as from strains from the TcI, TcII, TcIII and TcVI DTUs, based on nuclear and mitochondrial conserved genes to identify correlations among geographic and phylogenetic data. We also used SNP calling and Read Depth Coverage (RDC) analysis to estimate nuclear CCNV and mitochondrial heteroplasmy, revealing large divergences among TcII field isolates.

\section{Results}

To evaluate the genomic diversity among the T. cruzi TcII DTU within close geographic regions, we sequenced, de novo assembled and compared the nuclear and mitochondrial genome sequences of seven TcII field isolates. The assembly statistics for the nuclear genomes are available in the Additional file 1: Table S1. These parasites were isolated from chagasic patients from the central (S15 and S162a) and northeastern (S11, S23b, S44a, S92a, S154a,) regions of the Minas Gerais state, Brazil (Fig. 1a).

\section{T. cruzi nuclear and mitochondrial phylogeny}

From a total of 1,563 nuclear single copy genes that are conserved among the CL Brener Esmeraldo and Non-Esmeraldo-like haplotypes [28], 794 were partially de novo assembled in all seven TcII field isolates (Additional file 2: Table S2), while the other 769 genes were absent in at least one of the assemblies. From these 794, 701 genes were partially recovered after the Gblocks analysis (Additional file 3: Table S3), totalizing 558,587 nucleotides, which were used to estimate the nuclear maximum likelihood phylogeny of these strains. To better classify the TcII field samples, other strains from the DTUs TcI (Arequipa, Colombiana and Sylvio), TcII (Y strain and clones - Ycl2, Ycl4, Ycl6 - and Esmeraldo), TcIII (231), and TcVI (CL Brener Esmeraldo-like haplotype and CL Brener Non-Esmeraldo-like haplotype) were also included in this analysis (Fig. 1b). All the evaluated TcII strains/isolates clustered together and separated from the TcI and TcIII strains. As expected, the CL Brener (TcVI) Esmeraldo-like haplotype, which is derived from a TcII ancestor, clustered together with TcII strains. Similarly, the CL Brener (TcVI) Non-Esmeraldo-like haplotype, derived from a TcIII ancestor, clustered with the 231 (TcIII) strain. Concerning the TcII field isolates, two pair of samples, S15-S162a and S11-S92a, which were isolated from close geographic regions, were also clustered in the phylogenetic analysis, suggesting that for these isolates, genomic diversity correlates with geographic distances. On the other hand, the strains S11 and S154a that were isolated from the same locality had a distant phylogenetic relationship, suggesting that although geographic distance may correlate with TcII genomic diversity, there are different strains simultaneously coexisting in the same area (Fig. 1b). A principal component analysis (PCA) of all the differential nuclear SNPs found in the seven TcII field isolates also clustered together S15 - S162a and S11 - S92a and indicated S154a as a distantly-related isolate among the studied field samples (Fig. 1c).

Comparison of the gene conservation among the 19 newly assembled maxicircles, showed substantial differences in the kDNA sequences among $T$. cruzi DTUs (Fig. 2a). The CL Brener maxicircle shares higher identity (bit score $>40,000$ ) with sequences from Tulahuen, 231 and 9280; an intermediate identity (bit score $>19,000<$ 40,000 ) with TcI strains (Arequipa, Colombiana and Sylvio) and a lower identity (bit score $>4,000<19,000$ ) to TcII strains (S15, S23b, S44a, S154a, S162a, S11, S92a, Esmeraldo, Y clones and Y population) (Fig. 2a). Next, we compared the variability within TcII DTU kDNAs, using as root the Esmeraldo maxicircle (Fig. 2b). This analysis showed differences among TcII maxicircle sequences, where Esmeraldo sequence was closely related to the TcII field isolates, specially S44a, S154a and S23b (bit score > 37,000 ), and less similar to the $\mathrm{Y}$ clones and population (bit score $>18.000<37,000$ ). On the other hand, when only TcI maxicircles were evaluated, we found that they shared equally most of their sequences (Fig. 2c). Phylogenetic analysis of the 19 T. cruzi strains based on maxicircle coding genes separate the DTUs in a similar way to what was found with the nuclear phylogeny, with well-defined TcI and TcII clusters. Also, maxicircle analysis clustered $\mathrm{TcV}$ and $\mathrm{TcVI}$ strains closer to TcIII, reinforcing that the mitochondria from the hybrids DTUs $\mathrm{TcV}$ and $\mathrm{TcVI}$ were originated from the TcIII ancestor (Fig. 2d). As seen in the nuclear phylogeny, the two pairs of TcII strains from close geographic regions, S15-S162a and S11-S92a, also clustered in the maxicircle-derived phylogeny, while the strains from the same locality, S11 and S154a, had a distant phylogenetic relationship.

A comparative tanglegram between the nuclear and mitochondrial T. cruzi phylogeny showed large correspondences in most of the branches between both methodologies (Fig. 3), however, some discordances were also observed. Based on the maxicircle phylogeny, the sister group of the Y strain is the S11/S92a clade, while in the nuclear phylogeny, the Y sister group was S44a. The Esmeraldo sequence clustered with S154a in the nuclear based phylogeny, while clustered with S44a, but not with S154a, in the mitochondrial phylogeny. Finally, the four TcII isolates S15, S162a, S11 and S92a clustered together in the nuclear phylogeny but not in the maxicircle 


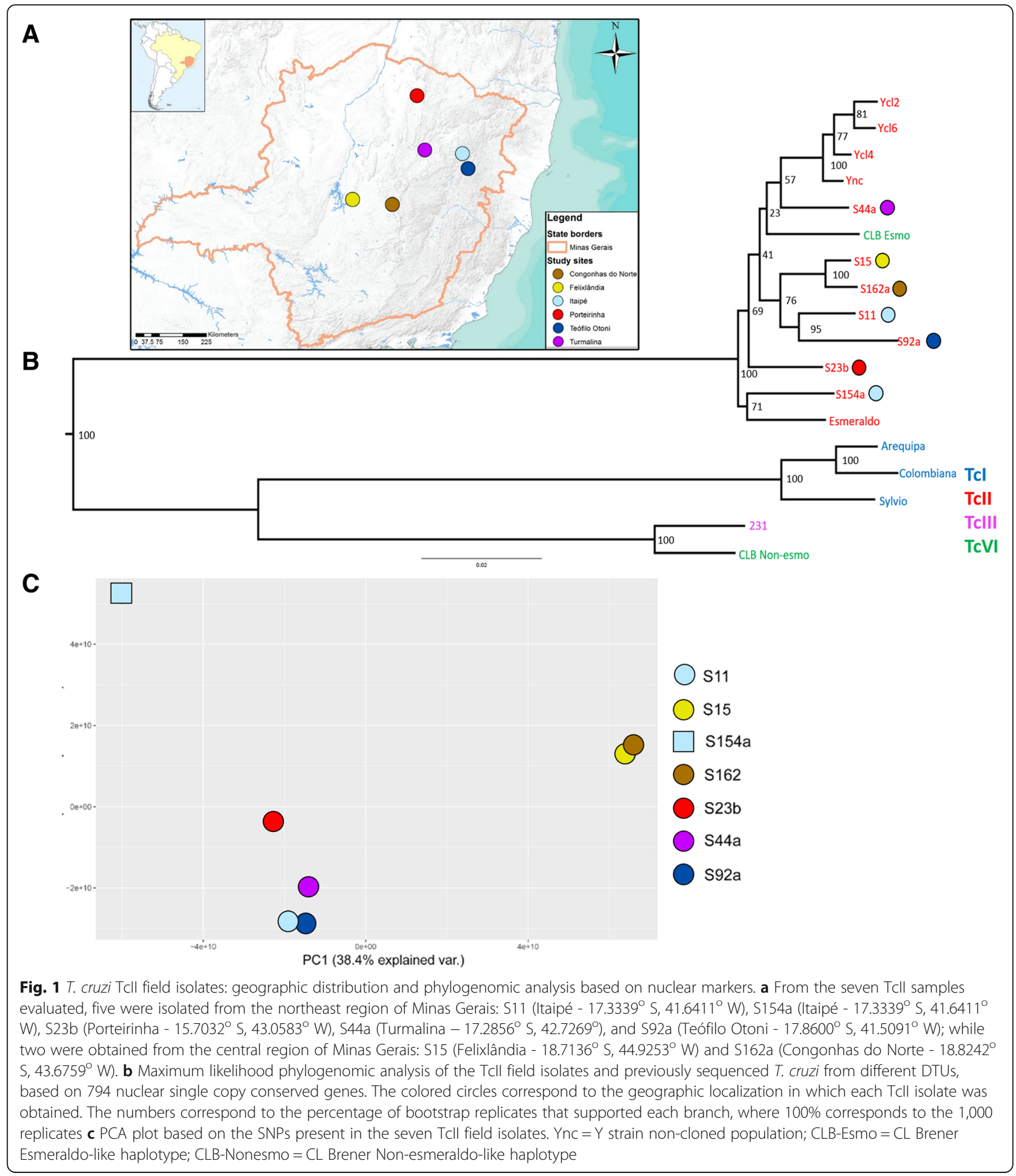

phylogeny. These discordances between nuclear and mitochondrial phylogeny could be caused by mitochondrial introgression events, where a parasite could inherit the mitochondria and nuclear genomes from different strains, resulting in discordant nuclear and mitochondrial phylogenies.
To search for evidences of mitochondrial heteroplasmy within TcII field isolates, we re-mapped the kDNA reads from each T. cruzi TcII sample in its reference-based assembled maxicircle sequence, and searched for heterozygous SNPs positions (Additional file 4: Figure S1). A total of 38, 46, 33, 29, 40,27, 36, 16, 17 and 21 

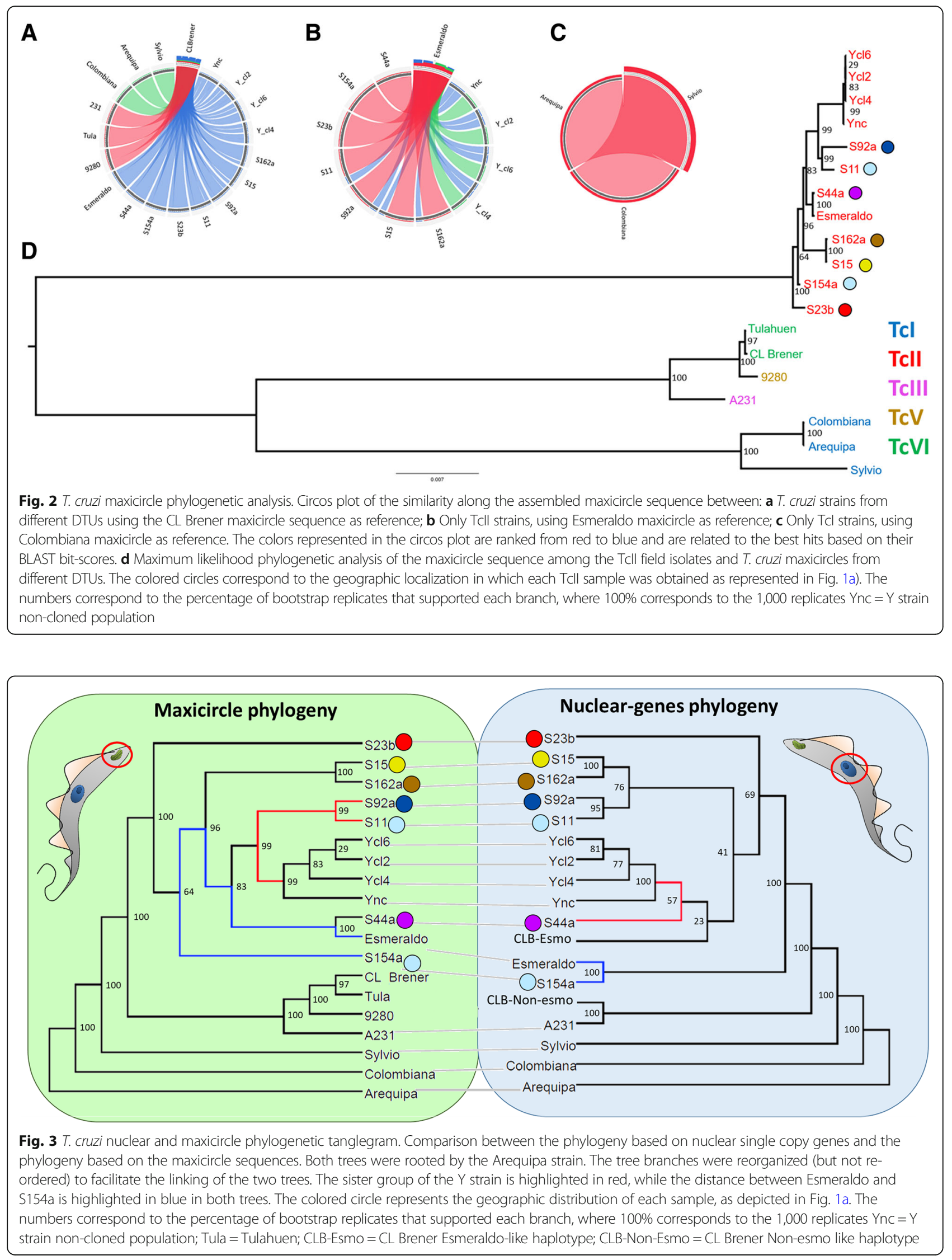
heterozygous SNPS were found, respectively, in the strains S11, S154a, S15, S162a, S23b, S44a, S92a, Ycl2, Ycl4, Ycl6 (Additional file 4: Figure S1A). The majority of these SNPs were localized in non-coding or repetitive regions (Additional file 4: Figure S1 B and C).

\section{Chromosomal copy number variation}

Analysis of chromosome copy number variation (CCNV) revealed large differences in the chromosomal duplication/loss events, highlighting the extensive ploidy variability found in this DTU (Fig. 4). The isolates S11, S154a and S162a presented more than 5 chromosomal duplication/losses, while S15 and S92 presented 5 and 4, and S23b and S44a had 3 or less aneuploidies with statistical significance (Fig. 4a, Additional file 5: Figure S2 and Additional file 6: Table S4). Interestingly, the phylogenetically close S15 and S162a isolates presented a similar chromosomal duplication/loss pattern, especially in the chromosomes 3, 7, 27 and 31 but a variable pattern in others, such as the chromosomes $6,13,22,34$, 38 and 39, suggesting that the duplication/loss of chromosomes is an ongoing process in T. cruzi evolution
(Fig. 4c). Similar results were obtained for the closely related S11 and S92 strains, which share expansions in the chromosomes 13 and 31, but not in the 6, 11, 21 and 27 chromosomes. To determine the overall genomic ploidy of each T. cruzi TcII field isolate, the allele frequency of heterozygous SNP in the whole genomes was estimated. The allele frequency distribution peak of 0.5 for all the strains reinforces the predominance of diploid chromosomes in T. cruzi (Fig. 4b).

To compare the CCNV pattern of the TcII field isolates with other DTUs, the chromosomal duplication/loss pattern in all $19 \mathrm{~T}$. cruzi strains evaluated by this work was estimated, showing several distinct patterns among and within DTUs, with some chromosomes being consistently duplicated or deleted (Fig. 5). The chromosome 31 was supernumerary in most of the strains from the five evaluated DTUs, while the chromosomes 6, 13, 27 also had an overall tendency to polyploidy, but in a lower extent than chromosome 31. There is also evidence for loss of chromosomes, as chromosomes 2,7 and 38 , which were in a haploid state in several T. cruzi strains. Next, to evaluate if the CCNV also vary within a given parasite population, we

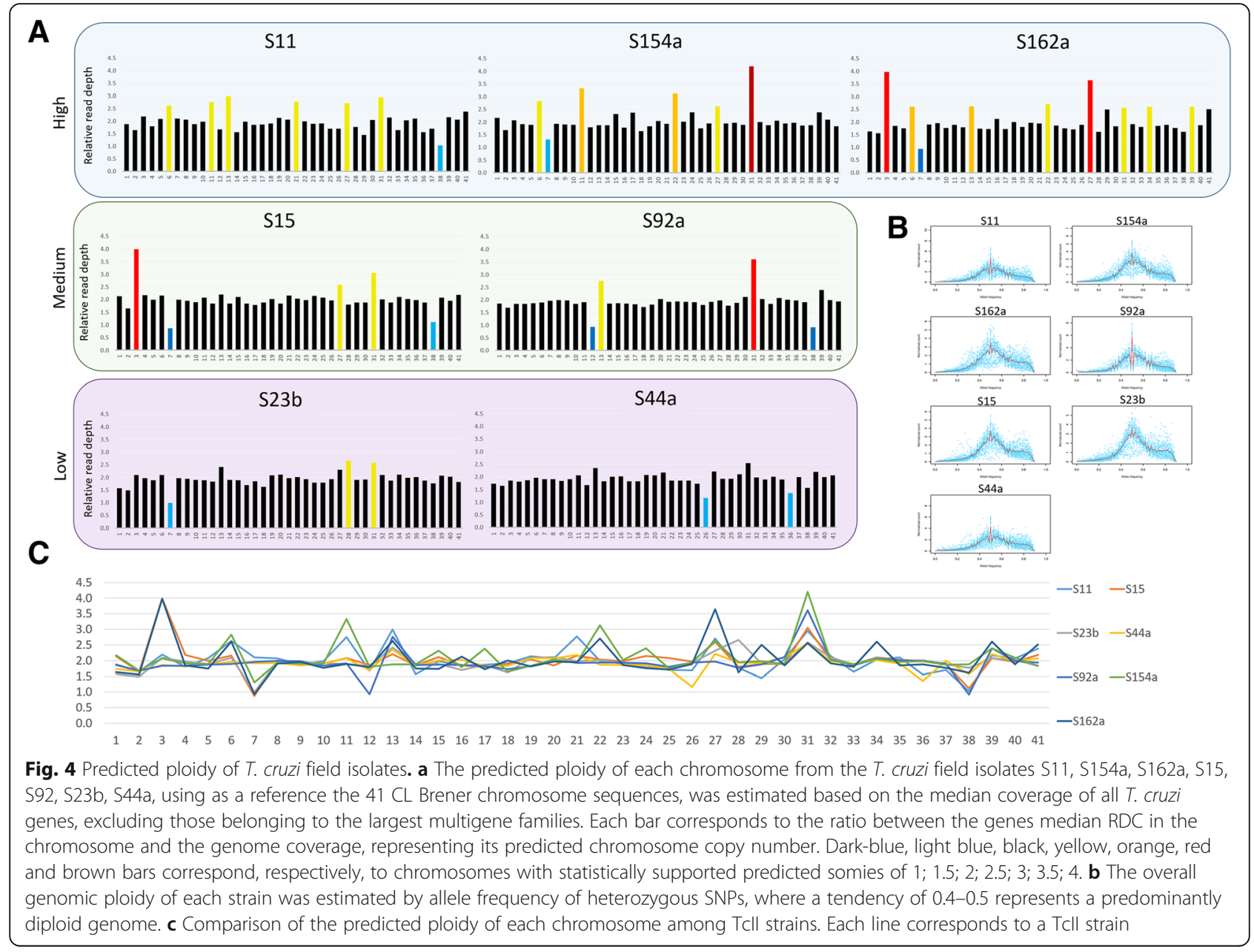




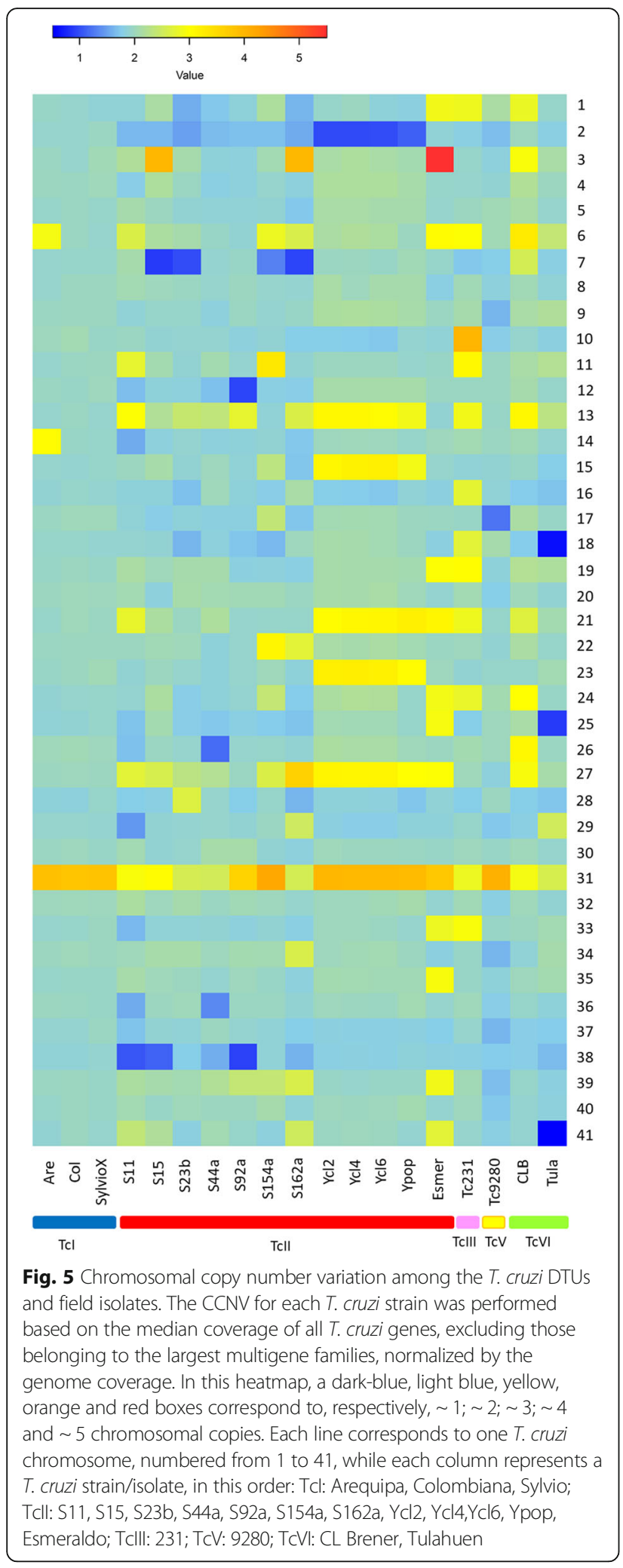

compared the chromosomal duplication/loss pattern in $Y$ strain and three clones derived from this strain: Ycl2, Ycl4 and Ycl6. All the Y clones had a similar pattern of CCNV with each other and with the $\mathrm{Y}$ strain (non-cloned population), indicating that the CCNV pattern may be constant within a population (Fig. 5). Interestingly, the Y strain chromosome 11 had a drastic change of RDC starting at the $248-\mathrm{kb}$ position, as seen in Reis-Cunha 2015 [28], resulting in an initial haploid and a terminal diploid state. To investigate if this difference is a result of mosaic aneuploidy in the population, where some cells have a complete chromosome 11 and others have a deletion of the initial $248 \mathrm{~kb}$, or if within individual cells one homolog chromosome have a complete and the other one a truncated copy, we compared RDC along the entire chromosome in the $\mathrm{Y}$ strain and clones. The initial $248 \mathrm{~kb}$ of the three $\mathrm{Y}$ clones presented half of the RDC from the remaining chromosomal sequence, in a similar way to what was found in the $Y$ strain (Additional file 7: Figure S3). This suggests that in individual parasites of $\mathrm{Y}$ strain, one copy of the $\mathrm{CL}$ Brener chromosome 11 is complete and the other one has an arm loss, resulting in a haploid state in its initial $248 \mathrm{~kb}$ sequence. Another possibility is that in $\mathrm{Y}$ strain, the corresponding CL Brener chromosome 11 is divided in two smaller chromosomes, a monosomic chromosome that correspond to the initial $248 \mathrm{~kb}$ and a disomic chromosome that corresponds to the remaining CL Brener chromosome 11 sequence.

A hierarchical clustering analysis based on the Euclidian distances of the predicted ploidy of each chromosome in the $19 \mathrm{~T}$. cruzi strains showed that the CCNV events do not follow the parasite phylogeny, as TcI, TcV and TcVI strains were clustered within TcII strains (Fig. 6). Interestingly, the Esmeraldo (TcII) and 231 (TcIII) strains presented the most divergent CCNV pattern among the evaluated strains/isolates. Sequencing of additional TcIII isolates are required to evaluate the extent of ploidy variation within this DTU. The TcII field isolates that were clustered together in the nuclear and mitochondrial phylogeny, S15-S162a and S11-S92a, also clustered together based on the CCNV profile.

\section{Discussion}

T. cruzi population genetics analysis are usually based on multilocus sequence typing of nuclear or mitochondrial markers, and therefore the comparison of the parasite variability is restricted to a number of genomic regions $[10,21,26,43-45]$. On the other hand, the use of next generation sequencing (NGS) reads provides a wide genomic characterization of the parasite variability, allowing not only a comparison based on a broader set of genes, but also the correlation of chromosomal amplification/loss patterns with the parasite phylogeny. Most of the T. cruzi molecular studies are focused on the TcI DTU, the oldest and 


\section{Cluster dendrogram with AU/BP values (\%)}

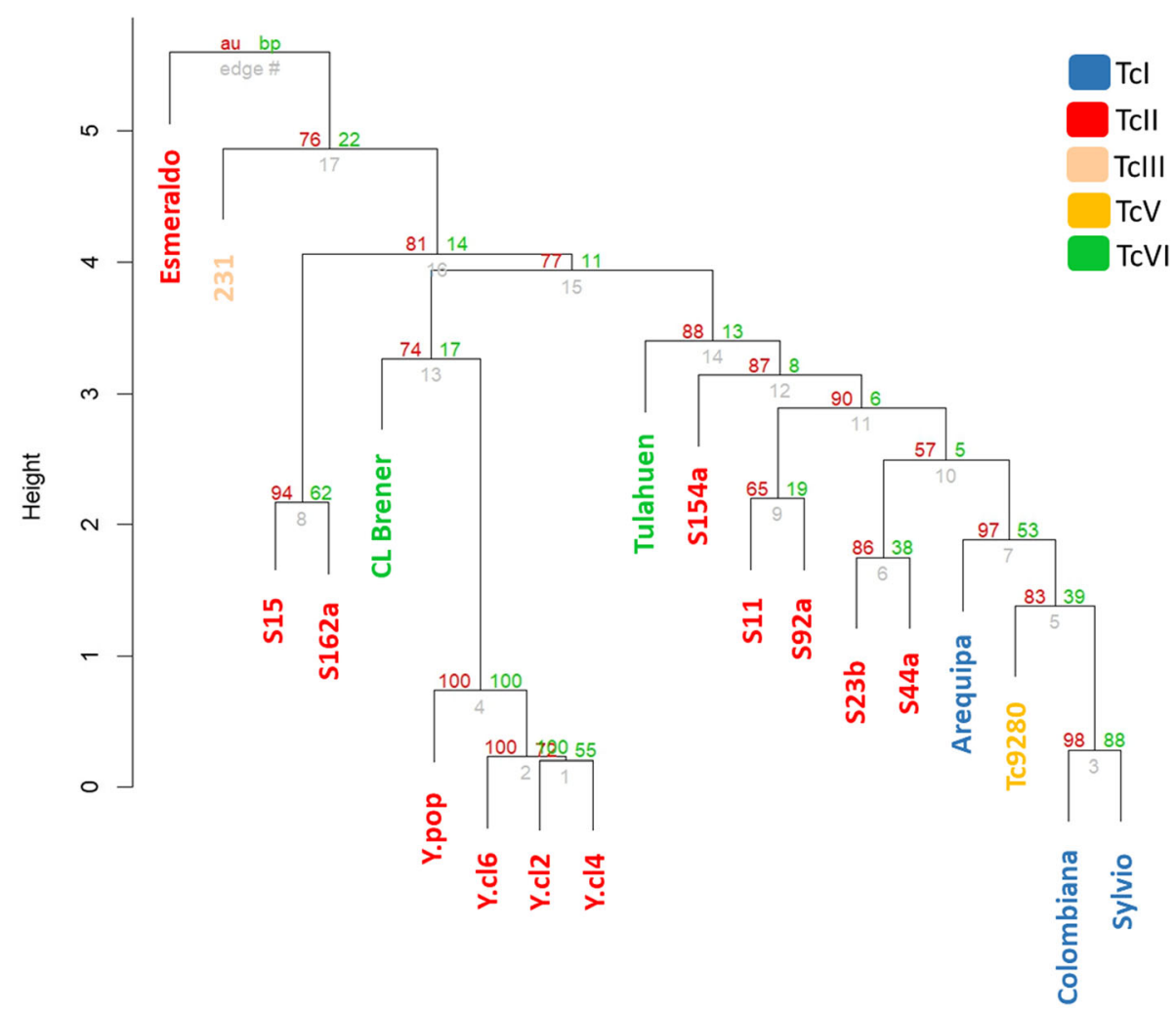

Distance: euclidean

Cluster method: complete

Fig. 6 Dendrogram of the hierarchical analysis based on T. cruzi chromosomal copy number variation patterns. The hierarchical clustering analysis based on Euclidean distances of the predicted ploidy of each chromosome of the 19 T. cruzi samples was performed using the R package Pvclust. Two bootstrap resampling methods were employed to assess the uncertainty in the hierarchical cluster analysis: approximately unbiased (au) in red and bootstrap probability (bp) in green

most widespread genetic lineage of the parasite, which is responsible for the majority of human infections from the Central America to Bolivia [5, 24, 26, 44-46]. There are, however, fewer studies concerning the variability, distribution and intercrossing of TcII, one of the most relevant $T$. cruzi subgroups related to human infection in the South America southern cone [5, 21, 47, 48]. Although the correspondence between DTU and clinical course is still unclear, some evidences point toward TcII association with severe manifestations of Chagas disease, presenting both cardiac and digestive manifestations, highlighting the importance of TcII for Chagas disease clinical outcomes $[5,24,49]$. To further explore the TcII variability, we sequenced the whole nuclear and mitochondrial genome of seven TcII isolated from patients from different locations in the Minas Gerais state, Brazil (Fig. 1a), and compared these isolates with each other and with reference strains from different DTUs. This is the first study to evaluate genome wide variation in $T$. cruzi isolates from close geographic locations, based on NGS.

\section{Nuclear and mitochondrial phylogeny}

The first step to estimate correlations among evolution, chromosomal duplication/loss and recombination within isolates from T. cruzi TcII DTU was the assessment of their phylogeny based on a set of nuclear conserved single copy genes as well as based on all mitochondrial genes [28]. Single copy genes are ideal molecular markers to infer phylogeny due to their uniqueness and conservation across and within species groups. Especially in analysis based on short Illumina reads, as de novo assembly or mapping of these small reads to repetitive regions (as microsatellites) could result in artefactual variations. However, the use of single copy genes prevents mapping errors and false SNPs that could compromise phylogenetic conclusions. Even though single-copy genes do not diverge as often as microsatellites, the use of a 
large dataset provided enough resolution to separate TcII from other DTUs and allowed inferences of phylogenetic proximity among these field samples (Fig. 1b). The simultaneous evaluation of nuclear and mitochondrial markers allows a thorough evaluation of a lineage evolutionary history, due to the different inheritance patterns and mutation rates presented by these two genomic sequences. T. cruzi hybrid strains present uniparental inheritance of its mitochondrial DNA and bi-parental inheritance of its nuclear genome, as seen in CL Brener (TcVI) where the mitochondria was originated from the TcIII ancestor and the nuclear genome is composed by sequences derived from TcII and TcIII ancestors [50, 51]. Besides, events of mitochondrial introgression and heteroplasmy have already been described in T. cruzi [26], which could be a consequence of intra or inter-DTU-hybridizations events.

A comparison of the phylogeny based on the nuclear single copy genes and the mitochondrial genes revealed that most of the branches are shared by the maximum likelihood phylogeny estimations by markers from both sequences (Fig. 3). The two samples from the central region of Minas Gerais, S15 and S162a, clustered together with high bootstrap value $(100 \%)$ in both nuclear and mitochondrial phylogenetic analysis as well as in the PCA plot based on whole genome SNPs (Figs. 1b, c and 2d). These two samples are separated from the other five TcII field isolates by the Espinhaço Mountain, a mountain range extending from the central region of Minas Gerais to the northern region of the Bahia State, Brazil. This geographic barrier could restrict the transit of insect vectors, separating $T$. cruzi populations from the central and north/northeastern regions of the state. The S154a sample was the outgroup of all TcII field samples based on the single copy genes phylogeny, clustering together with Esmeraldo (Fig. 1b) and presented the most divergent SNP pattern based on PCA analysis of whole nuclear genomic SNPs from the seven TcII field isolates (Fig. 1c). This suggests that S154a lineage could have endured several recombination events, being the most mosaic sample from the TcII isolates evaluated, or that it have diverged early from the other TcII field isolates. To date, the majority of field evidence supports that $T$. cruzi is not strictly clonal, and that recombination is a nonobligatory yet common event $[16,17,21,23,26,44]$. The occurrence of recombination among T. cruzi populations was documented in TcI from Bolivia [45, 46], Colombia [22] and Brazil [44], as well as among TcII strains from Brazil [21], based on samples from close geographic regions. T. cruzi strains were also capable of genetic recombination in laboratory, presenting fusion of parental genotypes, loss of alleles, homologous recombination and uniparental inheritance of kinetoplastid maxicircle genome [30]. Although T. cruzi-related parasites Leishmania sp. and T. brucei appears to undergo meiotic events in the insect vector [52-54], T. cruzi genetic exchange appears to occur in the mammalian host and is independent of an meiotic stage [16]. However, the rate in which these events occur in $T$. cruzi and whether recombination may also occur within insect vector is still unknown.

There were some divergences among the phylogeny of TcII field isolates, based on nuclear or mitochondrial genes (Fig. 3). The sister group of the Y clones/strain was S44a based on the nuclear single copy genes, and S11/S92a based on the mitochondrial genes (Fig. 3 - Red line). However, the bootstrap values supporting this nuclear branching was low. Similarly, Esmeraldo strain clustered with S154a based on nuclear markers and with S44a based on the maxicircle phylogeny (Fig. 3 - Blue line). This difference in the nuclear and maxicircle phylogeny are probably caused by mitochondrial introgression events, as recombination and gene exchange appears to be a common event in T. cruzi [20, 44]. Mitochondrial introgression was already been documented in some T. cruzi strains isolated from North and South America [10, 11, 25, 26]. Although the biological implications of mitochondrial introgression are still unknown, its occurrence reinforces the reliability of recombination inferences among $T$. cruzi strains. To search for mitochondrial heteroplasmy, the presence of heterogeneous mitochondrial genomes in an individual cell, we re-mapped the mitochondrial reads of each TcII strain to its reference-based maxicircle assembly and looked for heterozygous SNPs (Additional file 4: Figure S1). Only a few number of heterozygous SNPs were identified in the mitochondrial genome of TcII strains/isolates, and most of them were localized in repetitive regions, not supporting the occurrence of mitochondrial heteroplasmy. To date, reported levels of mitochondrial heteroplasmy in $T$. cruzi are scarce [26]. Heteroplasmy was already observed in the Sylvio X10/1 (TcI), based on re-mapping of its kDNA reads to the reference Sylvio X10/1 maxicircle, resulting in a total of 74 SNPs in eight genes and three intergenic regions [26]. In our analysis, we found only 1-3 heterozygous SNPs in coding genes, not providing enough support for heteroplasmy inferences. However, the absence strong evidence of heteroplasmy in our samples could be an underestimation resulting from low coverage $(\sim 60 \mathrm{X})$, when compared to $163 \mathrm{x}$ coverage used in the Sylvio X10/1 study [26], as only $12.2 \%$ of the reads corresponded to the minor variant SNPs in Sylvio [26].

\section{Chromosomal copy number variation}

Chromosomal copy number variation appears to be well tolerated in the trypanosomatids T. cruzi $[27,28]$ and Leishmania [55-61]. In the present work, we compared the CCNV pattern of seven TcII field samples with each other (Fig. 4), as well as with previously sequenced 
strains from the TcI, TcIII, TcV and TcVI DTUs (Fig. 5). It is well known that $T$. cruzi strains have distinct profiles of chromosomal bands based on Pulse Field Gel Electrophoresis analysis, and therefore a variable karyotype among DTUs. These differences where mainly attributed to expansion/retraction of multigene families clusters, or to events of chromosomal fusion/break during $T$. cruzi evolution [34, 51, 62-65]. Despite these variations, the housekeeping genes clusters are highly conserved and syntenic among the parasites strains and therefore represent an adequate source for sequence normalization in CCNV analysis [27, 28, 34, 66-68]. A hierarchical cluster dendrogram based on the CCNV pattern of the 19 T. cruzi strains from different DTUs grouped TcI, TcV and TcVI samples within TcII clusters, showing that the chromosomal duplication/loss events do not follow the phylogeny based on nuclear single copy genes or mitochondrial markers (Fig. 6). In fact, all the TcII strains evaluated had a different pattern of CCNV, with low (S23b, S44a) medium (S15, S92a) or high (S11, S154a, S162a) number of aneuploidies (Fig. 4), in accordance with which was previously observed between the TcII strains Esmeraldo and $Y$ [28]. This suggests that chromosomal gain/loss is frequent in T. cruzi, and occurs in a higher rate than DTU branching events, varying among and within DTUs $[27,28]$. The aneuploidy pattern also varies within close geographic populations of Leishmania donovani [55], reinforcing that both parasites are naturally aneuploids [69]. Based on FISH analysis, CCNV was identified within the same population in several Leishmania species and strains $[57,58,70]$. To explain this observation, a model based on miss segregation or stochastic replication of chromosomes was proposed in Leishmania [57, 58, 70]. In this model, there is an asymmetric replication and allotment of chromosomes during mitosis, resulting in polyploid and haploid cells. For this reason, the Leishmania population present a "mosaic aneuploidy", where cells from the same population presented different patterns of aneuploidies, and the most prevalent genotype within a population was estimated as $\sim 10 \%$ of the cell counts $[57,58]$. To evaluate if the aneuploidy pattern in $T$. cruzi also varies in a similar rate, we have cloned and sequenced the whole genome of three clones derived from Y strain, based on RDC. All the three Y clones as well as the Y strain [28] presented a similar aneuploidy pattern (Fig. 5), suggesting that although CCNV in T. cruzi varies among and within DTUs, it seems constant within a given population, different from what is observed in Leishmania. This data is in accordance with pulse field gel electrophoresis assays denoting that the chromosomal bands from the D11 clone from the G strain of T. cruzi was stable in continuous culture isolates over several years [34, 71].
Similarly, the $L$. donovani strain BPK282/0 cl4 presented a stable aneuploidy pattern for at least 32 passages after genome sequencing [55]. However, this unaltered pattern of aneuploidies could be a consequence of the normalized sum of the CCNV from the entire population, precluding the identification of aneuploidy patterns from single cells. In fact, several T. cruzi strains had estimated chromosomal ploidies with intermediated values (as between 2 and 3), which could be a consequence of a mixed cell population with disomic and trisomic chromosomes [28, 72]. A process that could explain the generation of polyploid cells in $T$. cruzi is the fusion of two parental diploid or polyploid cells followed by a progressive reduction of the chromosome number, in a similar way as the parasexual cycle of Candida albicans $[17,29,73]$. In this model, the fusion of 'parental' cells is followed by karyogamy and reductional mitotic division, which would lead to aneuploid daughter cells with different genomic/genetic contents [17, 58]. FACs analysis of T. cruzi hybrid isolates revealed an increase of $~ 70 \%$ in their DNA content when compared to parental strains [33]. The subsequent prolonged maintenance of experimental hybrids in axenic cultures lead to a gradual and progressive reduction in DNA content $[17,33]$, further supporting the parasexual model as basis to the generation of aneuploidies in $T$. cruzi.

Although structural variability and aneuploidies are usually associated with detrimental phenotypes in complex eukaryotes [74-76], some unicellular eukaryotes rely on aneuploidy as a mechanisms to allow rapid adaptation to changing environments, suggesting that the variation in chromosome number could also have a positive fitness effect in stress conditions [73, 77, 78]. Aneuploidy it is a common feature in trypanosomatids, described in several T. cruzi strains and Leishmania species $[28,55,56,79]$, however it appears to be absent in T. brucei [80]. As T. cruzi and Leishmania have their genome divided in a large number of fragments $(\sim 34$ to 47 putative chromosomes) [50, 51, 67, 81, 82], altering the copy number of specific chromosomes would alter the dosage of a restrict set of genes, avoiding detrimental consequences of large-scale dosage alterations. On the other hand, the diploid parasite T. brucei has its genome divided in eleven megabase-sized chromosomes [67, 81], suggesting that aneuploidies would be better supported in organisms that have its genome dispersed in a large number of chromosomes. However, the evaluation of a CCNV in a broader set of unicellular eukaryote species is necessary to confirm this hypothesis. Copy number variation is a well-documented mechanism to alter gene expression and enhance variability, especially in parasites that mostly regulate its gene expression post-transcriptionally as trypanosomatids [83-85]. Miss-segregation of chromosomes or the parasexual cycle could alter the copy number 
of several genes within a few generations, which may enable heteroxeneous parasites to rapidly adapt to the transition between the mammalian and invertebrate hosts $[17,60,61$, 72]. This hypothesis have been recently confirmed in Leishmania, where a shift in the pattern of duplicated/loss chromosomes was described as the parasite change from culture cells to insect vectors and to the mammalian host [61]. This shift in chromosome duplication patterns also impacted RNA levels, showing a higher expression of genes derived from polysomic chromosomes [61]. Alternatively, if a polysomic state is stable for long evolutionary periods it could allow the accumulation of mutations and consequent evolution of new functions for the duplicated genes, as the ancestral copy would still be present in the genome [72] The gain or loss of a whole chromosome was already associated with increased fitness in stress conditions and drug resistance in Saccharomyces cerevisiae, Candida albicans and carcinomatous lung cancer cells [77-79, 86], and could also be explored by the parasites to allow natural selection of favorable phenotypes. In fact, CCNV was also associated with drug resistance in L. major and L. infantum based on transcriptional profiling using microarrays, southern blot and comparative genomic hybridization, where these chromosomes reverted to disomy in the absence of drug pressure [79, 87]. However, Downing 2011, based on RDC analysis found no clear link between aneuploidy and drug resistance in $L$. donovani clinical isolates [55]. Drug selection also appears to promote gene amplification and translocation in T. cruzi [88], showing that genomic expansion is a widespread process employed by trypanosomatid parasites to survive to environmental changes.

The chromosome 31 was the only one supernumerary in the majority of the T. cruzi evaluated strains, been consistently polyploid among isolates from different DTUs (Fig. 5), as previously seen in a more restricted number of strains [28]. This chromosome is enriched with genes related to glycoprotein biosynthesis and glycosylation processes, especially with genes related to mucin glycosylation and biosynthesis, as the enzyme UDP-GlcNAc-dependent glycosyl-transferase [28, 89]. Mucins are highly glycosylated proteins that covers the whole surface of the parasite, which are directly involved in its survival in both invertebrate and vertebrate hosts $[89,90]$. One of the possible explanations for the expansion of chromosome 31 in $T$. cruzi could be the need to glycosylate the $2 \times 10^{6}$ mucins that covers the parasite surface $[28,89,90]$.

\section{Conclusions}

Next generation reads from whole genome and mitochondrial sequencing allows the simultaneous evaluation of phylogeny, aneuploidy and allele frequencies in the same population of cells, providing a genome-wide evaluation of the variability among closely geographic field isolates
$[28,55,69]$. Phylogenetic analysis of the TcII DTU suggested the occurrence of genomic recombination events during T. cruzi evolution in Minas Gerais, with possible mitochondrial introgression events. The discordance between the nuclear/mitochondrial phylogeny and the CCNV suggests that chromosomal gain/loss are more frequent than DTUs branching events in T. cruzi, and could be explored by the parasite to allow rapid selection of favorable phenotypes. Besides, the highly variable pattern of aneuploidies found within TcII field samples and the concordant pattern of CCNV within Y clones suggest that the parasexual cycle could be the major mechanism enrolled in genetic exchange and aneuploidy generation in geographically close $T$. cruzi isolates [17]. However, the miss segregation or stochastic replication of chromosomes, as proposed to Leishmania $[57,58]$, could also be a driving force in T. cruzi CCNV. To further address CCNV within a $T$. cruzi population, single-cell genome sequencing based analysis could provide a new level of resolution, comparing the whole chromosomal pattern of single parasites isolated from the same population. Aneuploidy constitutes a large source of adaptability, throughout gene dosage alterations and shaping of genetic heterogeneity [69], which could be important to the rapid adaptation and for the interchange between the invertebrate/mammal hosts in heteroxeneous parasites. Finally, the expansion of the chromosome 31 in a larger number of isolates/strains highlights the importance of the glycosylation to the $T$. cruzi survival.

\section{Methods}

\section{Genome sequencing and read libraries processing}

A total of $19 \mathrm{~T}$. cruzi whole genome sequencing read libraries containing samples from TcI, TcII, TcIII, TcV and TcVI DTUs were used in this study. Eleven of these sequences were generated in this work using Illumina Hiseq2000 sequencer, with $\sim 60 \mathrm{x}$ coverage, generating pair-end read libraries with $100 \mathrm{bp}$ read size and insert size of $350 \mathrm{bp}$. They consisted of seven TcII strains recently isolated from the central (S15 and S162a) and northeastern (S11, S23b, S44a, S92a and S154a) regions of Minas Gerais state, Brazil; three clones from the $Y$ strain (Ycl2, Ycl4, Ycl6); and one sample of the CL Brener (TcVI) strain. Other five T. cruzi whole genome and mitochondrial read libraries were generated by our group in a previous study [28] consisting of samples of TcI (Arequipa, Colombiana and Sylvio), TcII ( $\mathrm{Y}$ strain) and TcIII (231). The remaining three samples were downloaded from the National Center for Biotechnology Information Sequence Read Archive (NCBI-SRA), consisting of samples from the TcII (Esmeraldo), TcV (9280) and TcVI (Tulahuen) DTUs. The detailed description of each read library is summarized in the Additional file 8: Table S5. 
The quality of each read library was evaluated with the FASTQC tool (http://www.bioinformatics.babraham.ac.uk/projects/fastqc/) and filtered using Trimmomatic [91]. The phred filtering threshold was a minimum of 30 for Illumina reads and 20 for the 454 and Ion Torrent libraries, using a five nucleotide sliding window, as well as a minimum read size of 50 nucleotides.

The whole genome assembly contigs from all $\mathrm{CL}$ Brener Esmeraldo-like and Non-esmeraldo putative chromosomal sequences and unassigned contigs version 26 were downloaded from the TriTrypDB [92] (Additional file 9: Table S6).

\section{Parasite cloning and DNA isolation}

For cloning the T. cruzi Y (TcII) strain, $10^{3}$ epimastigotes were plated into a semi-solid medium (low-melting agarose $0.75 \%$, brain heart infusion $48.4 \%$, liver infusion tryptose (LIT) 48.4\%, 2.5\% defibrinated blood, and $250 \mu \mathrm{g} / \mathrm{mL}$ penicillin/streptomycin) and incubated at $28{ }^{\circ} \mathrm{C}$ for 35 days. Single clones were obtained and transferred to $25 \mathrm{~cm}^{3}$ culture flasks with $5 \mathrm{~mL}$ of LIT medium and 10\% fetal bovine serum. After cloning, the three Y clones (Ycl2, Ycl4 and Ycl6) epimastigote cultures where briefly cultured before DNA extraction. To isolate the parasite genomic and mitochondrial DNA, a total of $1 \times 10^{8} \mathrm{Y}$ epimastigotes were centrifuged at $3000 \mathrm{~g}$ for $10 \mathrm{~min}$ at $4{ }^{\circ} \mathrm{C}$. The parasites where washed three times with ice-cold PBS, suspended in PBS with $300 \mu \mathrm{g} / \mathrm{mL}$ proteinase $\mathrm{K}$ and incubated at $25{ }^{\circ} \mathrm{C}$ for $10 \mathrm{~min}$. The genomic DNA was obtained with the Wizard ${ }^{\circ}$ Genomic DNA Purification Kit (Promega), following the manufacturer instructions. The extracted DNA was submitted to a genotyping protocol using three different previously described markers to confirm the DTU identity [11, 93, 94].

\section{Nuclear genome assembly}

The genome assemblies of Esmeraldo, 231 and Sylvio strains, as well as the CL Brener Esmeraldo-like and Non-Esmeraldo haplotypes were downloaded, respectively, from the European Nucleotide Archive, NCBI and TriTrypDB (Links in the Additional file 9: Table S6). The genomes of the seven TcII field isolates, three $\mathrm{Y}$ clones, $\mathrm{Y}$ strain, Arequipa and Colombiana were de novo assembled, using Velvet optimizer with velvet version 1.2.10 [95, 96] for the Illumina, or using Celera 8.3 $[97,98]$ for the 454 read libraries. The NCBI accession numbers for the nuclear genome assemblies are listed in the Additional file 10: Table S7.

\section{kDNA assembly and sequence similarity visualization}

To select the most suitable mitochondrial sequence to be used in reference-based maxicircle assemblies, the read libraries for each of the T. cruzi strains were competitively mapped to all three publically available maxicircle references using BWA-mem [99, 100]. The available mitochondrial genomes with their respective NCBI accession numbers were: TcI Sylvio (FJ203996.1), TcII Esmeraldo (DQ343646.1) and TcVI CL Brener (DQ343645.1). The reference with the highest coverage for each strain was selected as a template. Based on this analysis, Sylvio maxicircle was selected as reference for Arequipa and Colombiana strains, Esmeraldo maxicircle was selected as reference for all the TcII field isolates, as well as for Y strain and clones and the CL Brener maxicircle was selected as reference for 231, 9280 and Tulahuen strains (Additional file 11: Figure S4). The final FASTA consensus maxicircle genome sequence was generated by submitting the BAM files to a pipeline using SAMTools mpileup, disabling probabilistic realignment for the computation of base alignment quality, reducing the chance of false SNPs caused by misalignments (-B), bcftools view using the minimum allele count of sites and including all sites with one or more genotypes $(-\mathrm{cg})$, vcfutils.pl to convert the bcftools vcf output file to a consensus fastq file (vcf2fq) and seqtk fq2fa to convert the fastq output to a final consensus fasta file [101]. The NCBI accession numbers for the maxicircle sequence assemblies obtained in this study are listed in the Additional file 10: Table S7. The maxicircle assemblies of the T. cruzi strains Sylvio (TcI), Esmeraldo (TcII) and $\mathrm{CL}$ Brener (TcVI) were downloaded from the aforementioned databases. To visualize the similarity patterns and differences between each one of the maxicircle sequences, a BLASTn search [102] between all samples with an e-value cutoff of $1 \mathrm{e}^{-20}$ was performed and submitted to Circoletto [103], a Circos program package [104].

\section{Phylogenetic analysis}

The nuclear phylogeny of 17 from the 19 T. cruzi samples was determined based on 1,563 CL Brener esmeraldo-like haplotype single copy nuclear genes described in Reis-Cunha 2015 [28]. These sequences were recovered from the assembled contigs of the aforementioned samples using BLAT [105], where only genes that were identified in all the assembled genomes where kept and used in the phylogenetic analysis. Tulahuen and 9280 strains were excluded from this analysis as their hybrid origin hampered the quality of the nuclear genome de novo assembly. For the kDNA phylogeny, all the 19 T. cruzi samples were used, including Tulahuen and 9280 . For both nuclear and mitochondrial genomes, each one of the recovered genes were aligned using MUSCLE [106] and the poorly aligned or gaps regions were eliminated using Gblocks [107]. The best fitting nucleotide substitution model for the phylogenetic 
analysis was determined using Jmodeltest [108]. The maximum likelihood phylogenetic tree was built using the PhyML [109], with the Generalized Time Reversible (GTR) model 1,000 bootstrap replicates, 0.9 proportion of invariable sites, 0.93 gamma distribution for the nuclear and 0.27 gamma distribution for the mitochondrial genome. The final phylogenetic tree images were built using FigTree v.1.4.2 software (http://tree.bio.ed.ac.uk/ software/figtree/). A comparative tanglegram based on the nuclear and mitochondrial markers were generated, using the program Dendroscope [110].

\section{Principal component analysis}

To estimate the distance among the seven TcII field isolates based on whole genome differential SNPs, a consensus nuclear genomic sequence was generated to each sample, using the GATK FastaAlternateReferenceMaker (https://software.broadinstitute.org/gatk/documentation/ tooldocs/current/org_broadinstitute_gatk_tools_walkers_fasta_FastaAlternateReferenceMaker.php). Then, a distance matrix based on differential SNPs was generated and loaded in the $\mathrm{R}$ caret package to generate the PCA plot (http://topepo.github.io/caret/index.html).

\section{Chromosomal copy number variation}

The estimation of the copy number of each chromosome from each strain was based on the median coverage of all genes present in a given chromosome excluding those that belong to the largest $T$. cruzi multigene families (trans-sialidase, MASP, TcMUC, RHS, DGF-1 and GP63) and the ones that had an outlier coverage based on Grubb's test. Briefly, T. cruzi CL Brener chromosomal reference sequences version 26 were downloaded from the TriTrypDB [92]. Then, the read libraries from the TcI and TcIII strains where mapped to the Non-Esmeraldo-like chromosomes, while strains from the TcII, TcV and TcVI were mapped to the Esmeraldo-like chromosomes [50] using BWA MEM [100]. The mapped reads were filtered by mapping quality 30 using SAMtools v1.1 [101], the RDC of each position in each chromosome was determined with BEDtools genomecov v2.16.2 [111] and in-house Perl scripts. For each chromosome, genes with outlier coverages were excluded, based on iterative Grubb's test, with $p<0.05$. The median RDC of all non-outlier genes in each chromosome was normalized by the genome coverage (estimated as the mean RDC of all single-copy genes in all chromosomes for each strain) and assumed as the chromosomal somy (Additional file 12: Figure S5A). Finally, the statistic support that a given chromosome somy was lower than $1 ; 1.5$ or higher than $2 ; 2.5 ; 3 ; 3.5 ; 4 ; 4.5$ or 5 was performed based on Mann-Whitney-Wilcoxon tests, with one-way analysis of variance and a significance of $p<0.05$, using R. A list containing all the genes used to estimate each chromosome somy of all seven TcII field isolates can be seen in the Additional file 13: Table S8.

Single-nucleotide polymorphisms (SNPs) of the mapped reads from all the T. cruzi strains were obtained using SAMtools mpileup function [101]. To be considered as a reliable SNP, the position RDC must be at least 10. For each chromosome, the proportion of read depth in alleles in each predicted heterozygous site was obtained and rounded to the second decimal place. Base frequencies were rounded in one hundred categories, ranging from 0.01 to 1 , and an approximate distribution of base frequencies for each chromosome was obtained by Perl scripts and plotted in R (www.r-project.org, R Development 2010) (Additional file 12: Figure S5B). To estimate the overall ploidy of each genome, the same methodology was applied, but the heterozygous positions from all CDSs from all chromosomes were employed simultaneously.

\section{Aneuploidy pattern dendrogram}

A hierarchical clustering analysis based on the predicted CCNV in all T. cruzi strains was performed with the $\mathrm{R}$ implemented Pvclust package [112]. A distance matrix was built with pairwise Euclidean distances between the strains, and the dendrogram was generated by complete linkage method. To assess the uncertainty in hierarchical clustering analysis, we used two bootstrap resampling methods implemented in Pvclust: bootstrap probability $(\mathrm{BP})$, the ordinary bootstrap resampling; and the approximately unbiased (AU) [113] probability, from multiscale bootstrap resampling. Both methods were calculated with 10,000 iterations.

\section{Additional files}

Additional file 1: Table S1. T. cruzi nuclear genome assembly statistics. (XLSX $10 \mathrm{~kb}$ )

Additional file 2: Table S2. T. cruzi CL Brener single-copy gene IDs from the 794 genes recovered from all genome assemblies. (XLSX 20 kb)

Additional file 3: Table S3. T. cruzi CL Brener single-copy gene IDs from the 701 genes used to estimate nuclear genomic Maximum Likelihood phylogeny. (XLSX 19 kb)

Additional file 4: Figure S1. Maxicircle heterozygous SNPs. To test for evidences of mitochondrial heteroplasmy, we evaluated the occurrence of heterozygous SNPs in the whole maxicircle sequence of all seven Tcll field isolates and three $Y$ clones. A) Total heterozygous SNP count in the maxicircle sequence. B) SNPs localized in the mitochondrial coding genes. C) SNPs distribution throughout the maxicircle sequence. In each box, the blue lines represent SNP positions, while the black line below corresponds to the whole maxicircle sequence, from 0 to $22,292 \mathrm{~kb}$. In this line, each coding gene is represented by a black box, and the repetitive region is represented by a red box. (DOCX $332 \mathrm{~kb}$ )

Additional file 5: Figure S2. Boxplot of the predicted ploidy of T. cruzi Tcll field isolates. The predicted ploidy of each chromosome from the $T$. cruzi field isolates S11, S15, S154a, S162a, S23b, S44a and S92a using as a reference the $41 \mathrm{CL}$ Brener chromosome sequences, was estimated based on the median coverage of all T. cruzi genes, excluding those belonging to the largest multigene families, and represented in boxplots. 
In this image, the predicted ploidy of each of the 41 chromosomes is represented by the median, first and third quartile, as well as maximum and minimum values. (A) Representation by strain. In this image, each quadrant corresponds to a Tcll strain, containing the predicted ploidy of all 41 chromosomes. (B) Representation by chromosome. In this image, each quadrant corresponds to a chromosome, comprising the predicted ploidy of this chromosome in all seven Tcll evaluated strains. (PPTX $4680 \mathrm{~kb}$ )

Additional file 6: Table S4. Ploidy estimations and statistic validation of all 41 chromosomes of the seven Tcll field isolates, S11, S15, S154a, S162a, S23b, S44a and S92a. The mean, median and standard deviation of the predicted ploidy of each chromosome of each strain, based on the coverage of all genes in a given chromosome is shown. The evaluation if the predicted ploidy of each chromosome was lower than $1 ; 1.5$ or higher than $2 ; 2.5 ; 3 ; 3.5 ; 4 ; 4.5$ or 5 was performed based on MannWhitney-Wilcoxon tests, with one-way analysis of variance and a significance of $p<0.05$, using R. Significant values are highlighted in red. (XLSX $46 \mathrm{~kb})$

Additional file 7: Figure S3. Read Depth Coverage of the chromosome 11 in the $Y$ strain and clones. In this picture, the blue lines correspond to the normalized RDC of each position of the chromosome 11, estimated by the ratio between the RDC and the genome coverage. The red line corresponds to the $248 \mathrm{~kb}$ position in the chromosome. Below, the protein-coding genes are depicted as rectangles drawn as proportional to their length, and their coding strand is indicated by their position above (top strand) or below (bottom strand) the central line. Cyan and black rectangles represent multigene families and hypothetical/housekeeping genes, respectively. The initial 248-kb in this chromosome had a smaller RDC when compared to remaining sequence in the $Y$ strain as well as in all three $Y$ clones evaluated. (DOCX $226 \mathrm{~kb}$ )

Additional file 8: Table S5. T. cruzi read libraries description. (DOCX 14 kb) Additional file 9: Table S6. Links to download T. cruzi reference genomes. (DOCX $12 \mathrm{~kb}$ )

Additional file 10: Table S7. NCBI accession numbers of the T. cruzi genomes and maxicircle assemblies. (DOCX $14 \mathrm{~kb}$ )

Additional file 11: Figure S4. Competitive mapping of the mitochondrial reads to the three available maxicircle templates. The percentage of mitochondrial genome reads from the $16 \mathrm{~T}$. cruzi read libraries that mapped preferentially with each of the maxicircle sequence templates, Sylvio (TCl), Esmeraldo (TCII) and CL Brener (TcVI with mitochondria sequence derived from TCIII) is shown. The Tcl strains mapped preferentially with the Sylvio template, while Tcll strains mapped preferentially with the $Y$ strain and the Tclll, $V$ and $V I$ strains mapped preferentially with the $\mathrm{CL}$ Brener maxicircle sequence. (DOCX $235 \mathrm{~kb}$ )

Additional file 12: Figure S5. Methodology for T. cruzi CCNV estimations. (A) The CCNV estimations were performed using the median coverage of all $T$. cruzi genes, excluding those belonging to the largest multigene families in each one of the $\mathrm{CL}$ Brener 41 putative chromosomes as an estimate of its chromosome copy number. In brief, the median RDC of the selected genes in each of the $41 \mathrm{CL}$ Brener chromosomes were generated by PERL scripts and normalized by the genome coverage. The genome coverage was estimated as the mean $\mathrm{RDC}$ of all single-copy genes in all chromosomes for each strain. (B) Heterozygous SNPs between the $\mathrm{CL}$ Brener chromosome and the mapped reads for the $T$. cruzi stains were obtained from the filtered SAMtools mpileup results. To be considered as a reliable SNP, the position RDC must be at least 10, with 5 reads supporting each variant. For each chromosome, the proportion of the alleles in each predicted heterozygous site was obtained and rounded to the second place. Base frequencies were rounded in ten categories, ranging from 0.01 to 1.00 , and an approximate distribution of base frequencies for each chromosome was plotted in $\mathrm{R}$. Disomic chromosomes have a peak in 0.50 , while trisomic chromosomes have peaks in 0.33 and 0.66 . Tetrasomic chromosomes have combination of peaks of $0.20,0.80$ and 0.50 . (DOCX $120 \mathrm{~kb}$ )

Additional file 13: Table S8. List of genes used to estimate the chromosomal ploidy of the seven Tcll field isolates, after the exclusion of genes with outlier coverages based on iterative Grubbs' tests. Each isolate, S11, S154a, S162a, S15, S92a, S23b and S44a is represented in a different sheet. (XLSX $1150 \mathrm{~kb}$ )

\section{Acknowledgements}

We thank Vitor Borda for his support in the population structure analysis and Michele Silva de Matos for the technical support.

\section{Funding}

This study was funded by Fundação de Amparo a Pesquisa do Estado de Minas Gerais (FAPEMIG), Instituto Nacional de Ciência e Tecnologia de Vacinas (INCTV) - Conselho Nacional de Desenvolvimento Científico e Tecnológico (CNPq), Coordenação de Aperfeiçoamento de Pessoal de Nível Superior (CAPES), Pró-reitoria de Pesquisa, Universidade Federal de Minas Gerais. DCB and EC are CNPq research fellows. JLRC, GFRL and ACS received scholarships from CAPES; RPB and LVA received a scholarship from CNPq

\section{Availability of data and materials}

Read files are available through the NCBI Sequence Read Archive (SRA), under the project number PRJNA421475. The read library data is further described in the Additional file 8: Table S5.

\section{Authors' contributions}

JLRC conceived the design of the study, performed all analyses, and draft the manuscript; RPB performed the maxicircle assemblies and phylogeny analyses; GFRL, ACS, RTF and HRV, performed PCA analysis and participated in the chromosome copy number estimation analysis; LVA and MSC cloned the $Y$ strain; RPB, GFRL, ACS, HOV, LVA, MSC, FHCD, RTF, DAD, LMCG and EC participated in the study design and helped to draft the manuscript; $D C B$ and GCC conceived the study, its design and coordination and wrote the manuscript. All authors read and approved the final manuscript.

\section{Ethics approval and consent to participate}

T. cruzi isolation from patients and all procedures were performed with the informed consent of the participants and approved by the Ethics Committee 087/99 of UFMG, Belo Horizonte, MG, Brazil.

\section{Consent for publication}

Not applicable.

\section{Competing interests}

Daniella C. Bartholomeu is a member of the BMC Genomics Editorial Board.

\section{Publisher's Note}

Springer Nature remains neutral with regard to jurisdictional claims in published maps and institutional affiliations.

\section{Author details}

'Departamento de Parasitologia, Universidade Federal de Minas Gerais, Belo Horizonte, Brazil. ${ }^{2}$ The University of Georgia, Athens, USA. ${ }^{3}$ Departamento de Microbiologia, Imunologia e Parasitologia, Universidade Federal de Santa Catarina, Florianópolis, Brazil. ${ }^{4}$ U.S. Naval Medical Research, Lima, Peru. ${ }^{5}$ Broad Institute of MIT and Harvard, Boston, USA.

Received: 19 December 2017 Accepted: 23 October 2018

Published online: 13 November 2018

\section{References}

1. Hotez PJ, Bottazzi ME, Franco-Paredes C, Ault SK, Periago MR. The neglected tropical diseases of Latin America and the Caribbean: a review of disease burden and distribution and a roadmap for control and elimination. PLoS Negl Trop Dis. 2008;2.

2. Coura JR, Borges-Pereira J. Chagas disease: what is known and what should be improved: a systemic review. Rev Soc Bras Med Trop. 2012;45:286-96.

3. WHO. Research priorities for Chagas disease, human African trypanosomiasis and leishmaniasis. World Health Organ Tech Rep Ser. 2012;v-xii, 1-100. Available from: http://www.ncbi.nlm.nih.gov/pubmed/23484340

4. Zingales B, Andrade SG, Briones MRS, Campbell DA, Chiari E, Fernandes O, et al. A new consensus for Trypanosoma cruzi intraspecific nomenclature: second revision meeting recommends Tcl to TcVI. Mem Inst Oswaldo Cruz. 2009;104:1051-4.

5. Zingales B, Miles MA, Campbell DA, Tibayrenc M, Macedo AM, Teixeira MMG, et al. The revised Trypanosoma cruzi subspecific nomenclature: Rationale, epidemiological relevance and research applications. Infect Genet 
Evol. 2012;12:240-53 Available from: https://doi.org/10.1016/j.meegid.2011. 12.009.

6. Pinto CM, Kalko EKV, Cottontail I, Wellinghausen N, Cottontail VM. TcBat a bat-exclusive lineage of Trypanosoma cruzi in the Panama Canal zone, with comments on its classification and the use of the 18S rRNA gene for lineage identification. Infect Genet Evol. 2012;12:1328-32.

7. Barnabé C, De Meeûs T, Noireau F, Bosseno MF, Monje EM, Renaud F, et al. Trypanosoma cruzi discrete typing units (DTUs): microsatellite loci and population genetics of DTUs TCV and Tcl in Bolivia and Peru. Infect Genet Evol. 2011;11:1752-60

8. del P Fernández M, Cecere MC, Lanati LA, Lauricella MA, Schijman AG, Gürtler RE, et al. Geographic variation of Trypanosoma cruzi discrete typing units from Triatoma infestans at different spatial scales. Acta Trop. 2014;140: 10-8 Available from: https://doi.org/10.1016/j.actatropica.2014.07.014.

9. Lima VDS, Xavier S, das Cristina C, Maldonado I, Fabiola R, Rodrigues RAL, Vicente A, Carolina P, Maria JA. Expanding the knowledge of the geographic distribution of Trypanosoma cruzi Tcll and TcV/TcVl genotypes in the Brazilian Amazon. PLoS One. 2014;9:e116137.

10. Messenger LA, Ramirez JD, Llewellyn MS, Guhl F, Miles MA. Importation of hybrid human-associated Trypanosoma cruzi strains of southern south American origin. Colombia Emerg Infect Dis. 2016;22:1452-5.

11. De Freitas JM, Augusto-Pinto L, Pimenta JR, Bastos-Rodrigues L, Gonçalves VF, Teixeira SMR, et al. Ancestral genomes, sex, and the population structure of Trypanosoma cruzi. PLoS Pathog. 2006;2:0226-35.

12. Westenberger SJ, Barnabé C, Campbell DA, Sturm NR. Two hybridization events define the population structure of Trypanosoma cruzi. Genetics. 2005; 171:527-43.

13. Burgos JM, Risso MG, Brenière SF, Barnabé C, Campetella O, Leguizamón MS. Differential distribution of genes encoding the virulence factor trans-Sialidase along Trypanosoma cruzi discrete typing units. PLoS One. 2013;8:9-11.

14. Tomasini N, Diosque P. Evolution of Trypanosoma cruzi: clarifying hybridisations, mitochondrial introgressions and phylogenetic relationships between major lineages. Mem Inst Oswaldo Cruz. 2015;110:403-13.

15. Machado CA, Flores-lo CA. Analyses of 32 loci clarify phylogenetic relationships among Trypanosoma cruzi lineages and support a single hybridization prior to human. Contact Dermatitis. 2011;5.

16. Lewis MD, Llewellyn MS, Yeo M, Acosta N, Gaunt MW, Miles MA. Recent, independent and anthropogenic origins of Trypanosoma cruzi hybrids. PLoS Negl Trop Dis. 2011;5.

17. Messenger LA, Miles MA. Evidence and importance of genetic exchange among field populations of Trypanosoma cruzi. Acta Trop. 2015;151:150-5.

18. Tibayrenc M, Kjellberg F. A clonal theory of parasitic protozoa : The population structures of Trichomonas, and Trypanosoma and their medical and taxonomical consequences. Proc Natl Acad Sci U S A. 1990;87:2414-8.

19. Tibayrenc M, Ayala FJ. Is predominant clonal evolution a common evolutionary adaptation to parasitism in pathogenic parasitic Protozoa Fungi, Bacteria, and viruses? [internet]. Adv Parasitol. Elsevier Ltd; 2016. Available from: https://doi.org/10.1016/bs.apar.2016.08.007

20. Tibayrenc M, Ayala FJ. How clonal are Trypanosoma and Leishmania. Trends Parasitol. [Internet]. Elsevier Ltd. 2012:1-6 Available from: https://doi.org/10. 1016/j.pt.2013.03.007.

21. Baptista R de P, D'Avila DA, Segatto M, Valle ÍF do, Franco GR, Valadares HMS, et al. Evidence of substantial recombination among Trypanosoma cruzi II strains from Minas Gerais. Infect Genet Evol. [Internet]. Elsevier B.V.; 2014; 22:183-191. Available from: https://doi.org/10.1016/j.meegid.2013.11.021

22. Ramírez JD, Guhl F, Messenger LA, Lewis MD, Montilla M, Cucunuba Z, et al. Contemporary cryptic sexuality in Trypanosoma cruzi. Mol Ecol. 2012;21:4216-26.

23. Ramírez JD, Llewellyn MS. Reproductive clonality in protozoan pathogens-truth or artefact? Mol Ecol. [internet]. 2014;23:4195-4202. Available from: http://www.ncbi.nlm.nih.gov/pubmed/25060834.

24. Messenger LA, Miles MA, Bern C. Between a bug and a hard place: Trypanosoma cruzi genetic diversity and the clinical outcomes of Chagas disease. Expert Rev Anti Infect Ther. 2015;13:995-1029 Available from: http:// www.pubmedcentral.nih.gov/articlerender.fcgi?artid=4784490\&tool= pmcentrez\&rendertype $=$ abstract.

25. Machado $\mathrm{C}$ a, Ayala FJ. Nucleotide sequences provide evidence of genetic exchange among distantly related lineages of Trypanosoma cruzi. Proc Nat Acad Sci USA. 2001;98:7396-401.

26. Messenger LA, Llewellyn MS, Bhattacharyya T, Franzén O, Lewis MD, Ramírez $J \mathrm{D}$, et al. Multiple mitochondrial introgression events and heteroplasmy in
Trypanosoma cruzi revealed by maxicircle MLST and next generation sequencing. PLoS Negl Trop Dis. 2012;6.

27. Minning T a, Weatherly DB, Flibotte S, Tarleton RL. Widespread, focal copy number variations (CNV) and whole chromosome aneuploidies in Trypanosoma cruzi strains revealed by array comparative genomic hybridization. BMC Genomics. 2011;12:139 Available from: http://www. biomedcentral.com/1471-2164/12/139.

28. Reis-Cunha JL, Rodrigues-Luiz GF, Valdivia HO, Baptista RP, Mendes TAO, de Morais GL, et al. Chromosomal copy number variation reveals differential levels of genomic plasticity in distinct Trypanosoma cruzi strains. BMC Genomics. 2015;16:499 Available from: http://www.biomedcentral.com/ 1471-2164/16/499.

29. Bennett RJ. The parasexual lifestyle of Candida albicans. Curr Opin Microbiol. 2015;28:10-7 Available from: https://doi.org/10.1016/j.mib.2015.06.017.

30. Gaunt MW, Yeo M, Frame IA, Stothard JR, Carrasco HJHJ, Taylor MC, et al. Mechanism of genetic exchange in American trypanosomes. Nature. 2003; 421:936-9.

31. Heitman J. Sexual Reproduction and the Evolution of Microbial Pathogens Review. Curr Biol. 2006;16:711-25.

32. Sturm NR, Campbell DA. Alternative lifestyles: The population structure of Trypanosoma cruzi. Acta Trop. 2010;115:35-43 Available from: https://doi. org/10.1016/j.actatropica.2009.08.018.

33. Lewis MD, Llewellyn MS, Gaunt MW, Yeo M, Carrasco HJ, Miles MA. Flow cytometric analysis and microsatellite genotyping reveal extensive DNA content variation in Trypanosoma cruzi populations and expose contrasts between natural and experimental hybrids. Int J Parasitol ; 2009;39:13051317. Available from: https://doi.org/10.1016/j.jpara.2009.04.001

34. Souza RT, Lima FM, Barros RM, Cortez DR, Santos MF, Cordero EM, et al. Genome size, karyotype polymorphism and chromosomal evolution in Trypanosoma cruzi. PLoS One. 2011;6.

35. Lukes J, Guilbride DL, Voty J, Zíkova A, Benne R, Englund PT. MINIREVIEW Kinetoplast DNA Network : Evolution of an Improbable Structure. Eukaryot Cell. 2002;1:495-502.

36. Aphasizheva I, Aphasizhev R. U-insertion/deletion mRNA-editing holoenzyme: definition in sight. Trends Parasitol. 2016;32:144-56 Available from: https://doi.org/10.1016/.jpt.2015.10.004.

37. Feagin JE. RNA editing in kinetoplastid mitochondria. J Biol Chem. 1990;265:19373-6.

38. Landweber LF. The evolution of RNA editing in kinetoplastid protozoa. Biosystems. 1992;28:41-5.

39. Stuart K. RNA editing in kinetoplastid protozoa. Curr Opin Genet Dev. 1991; 1:412-6.

40. Telleria J, Lafay B, Virreira M, Barnabé C, Tibayrenc M, Svoboda M. Trypanosoma cruzi : Sequence analysis of the variable region of kinetoplast minicircles. Exp Parasitol. 2006;114:279-88.

41. Carranza JC, Valadares HMS, D'Ávila DA, Baptista RP, Moreno M, Galvão LMC, et al. Trypanosoma cruzi maxicircle heterogeneity in Chagas disease patients from Brazil. Int J Parasitol 2009;39:963-973. Available from: https://doi.org/ 10.1016/j.jpara.2009.01.009

42. Miles MA, Llewellyn MS, Lewis MD, Yeo M, Baleela R, Fitzpatrick S, et al. The molecular epidemiology and phylogeography of Trypanosoma cruzi and parallel research on Leishmania: looking back and to the future. Parasitology. 2009;136:1509-1528. Available from: http://www.ncbi.nlm.nih. gov/pubmed/19691868.

43. Llewellyn MS, Miles MA, Carrasco HJ, Lewis MD, Yeo M, Vargas J, et al. Genome-scale multilocus microsatellite typing of Trypanosoma cruzi discrete typing unit I reveals phylogeographic structure and specific genotypes linked to human infection. PLoS Pathog. 2009;5.

44. Lima VS, Jansen AM, Messenger $L$ a, Miles $M$ a, Llewellyn MS. Wild Trypanosoma cruzi I genetic diversity in Brazil suggests admixture and disturbance in parasite populations from the Atlantic Forest region. Parasit Vectors [Internet]. 2014;7:263. Available from: http://www. pubmedcentral.nih.gov/articlerender.fcgi?artid=4062772\&tool= pmcentrez\&rendertype $=$ abstract

45. Messenger LA, Garcia L, Vanhove M, Huaranca C, Bustamante M, Torrico M, et al. Ecological host fitting of Trypanosoma cruzi Tcl in Bolivia: mosaic population structure, hybridization and a role for humans in Andean parasite dispersal. Mol Ecol. 2015;24:2406-22.

46. Barnabe C, Buitrago R, Bremond P, Aliaga C, Salas R, Vidaurre P, et al. Putative panmixia in restricted populations of Trypanosoma cruzi isolated from wild triatoma infestans in Bolivia. PLoS One. 2013;8. 
47. D'Ávila DA, Macedo AM, Valadares HMS, Gontijo ED, De Castro AM, Machado CR, et al. Probing population dynamics of Trypanosoma cruz during progression of the chronic phase in chagasic patients. J Clin Microbiol. 2009;47:1718-25.

48. Da Câmara ACJ, Lages-Silva E, Sampaio GHF, D'Ávila DA, Chiari E, Da Cunha Galvão LM. Homogeneity of Trypanosoma cruzi I, II, and III populations and the overlap of wild and domestic transmission cycles by Triatoma brasiliensis in northeastern Brazil. Parasitol Res. 2013;112:1543-50.

49. Luquetti AO, Miles MA, Rassi A, de Rezende JM, de Souza AA, Povoa MM, et al. Trypanosoma cruzi: zymodemes associated with acute and chronic Chagas' disease in central Brazil. Trans R Soc Trop Med Hyg [Internet]. 1986;80:462-470. Available from: http://www.ncbi.nlm.nih. gov/entrez/query.fcgi?cmd=Retrieve\&db=PubMed\&dopt=Citation\&list_ uids $=3099437$

50. Weatherly DB, Boehlke C, Tarleton RL. Chromosome level assembly of the hybrid Trypanosoma cruzi genome. BMC Genomics. 2009;10:255.

51. El-Sayed NM. The genome sequence of Trypanosoma cruzi, etiologic agent of chagas disease. Science [Internet]. 2005;309:409-15. Available from: http://www.sciencemag.org/cgi/doi/10.1126/science.1112631

52. Peacock L, Ferris V, Sharma R, Sunter J, Bailey M, Carrington M, et al. Identification of the meiotic life cycle stage of Trypanosoma brucei in the tsetse fly. Proc Natl Acad Sci USA. [Internet]. 2011;108:3671-3676. Available from: http://www.pnas.org/content/108/9/3671.abstract

53. Peacock L, Bailey M, Carrington M, Gibson W. Meiosis and haploid gametes in the pathogen Trypanosoma brucei. Curr Biol. 2014;24:181-6 Available from: https://doi.org/10.1016/j.cub.2013.11.044.

54. Rogers MB, Downing T, Smith BA, Imamura H, Sanders M, Svobodova M, et al. Genomic confirmation of hybridisation and recent inbreeding in a vector-isolated Leishmania population. PLoS Genet. 2014;10.

55. Downing T, Imamura H, Decuypere S, Clark TG, Coombs GH, Cotton J a., et al. Whole genome sequencing of multiple Leishmania donovani clinical isolates provides insights into population structure and mechanisms of drug resistance. Genome Res. [Internet]. 2011;21:2143-2156. Available from: https://doi.org/10.1101/gr.123430.111

56. Rogers MB, Hilley JD, Dickens NJ, Wilkes J, Bates P a, Depledge DP, et al. Chromosome and gene copy number variation allow major structural change between species and strains of Leishmania. Genome Res. 2011;21:2129-42.

57. Sterkers Y, Lachaud L, Crobu L, Bastien P, Pagès M. FISH analysis reveals aneuploidy and continual generation of chromosomal mosaicism in Leishmania major. Cell Microbiol. 2011;13:274-83.

58. Sterkers Y, Crobu L, Lachaud L, Pagès M, Bastien P. Parasexuality and mosaic aneuploidy in Leishmania: alternative genetics. Trends Parasitol. 2014;30:429-35.

59. Valdivia HO, Reis-Cunha JL, Rodrigues-Luiz GF, Baptista RP, Baldeviano GC, Gerbasi R V., et al. Comparative genomic analysis of Leishmania (Viannia) peruviana and Leishmania (Viannia) braziliensis. BMC Genomics [Internet]. 2015;16:715. Available from: http://www.biomedcentral.com/ $1471-2164 / 16 / 715$

60. Reis-Cunha JL, Valdivia HO, Bartholomeu DC. Trypanosomatid Genome Organization and Ploidy. In: Silva MS, Cano MI, editors. Mol. Cell. Biol. Pathog. Trypanos. 1st ed Frontiers in Parasitology; 2017. p. 61-103.

61. Dumetz F, Imamura H, Sanders M, Seblova V, Myskova J, Pescher P. Modulation of aneuploidy in Leishmania donovani during adaptation to different in vitro and in vivo environments and its impact on gene expression. MBio. 2017:8:1-14.

62. Vargas N, Pedroso A, Zingales B. Chromosomal polymorphism, gene synteny and genome size in T. cruzi I and T. cruzi II groups. Mol Biochem Parasitol. 2004;138:131-41.

63. Pedroso A, Cupolillo E, Zingales B. Evaluation of Trypanosoma cruzi hybrid stocks based on chromosomal size variation. Mol Biochem Parasitol. 2003; 129:79-90.

64. Triana O, Ortiz S, Dujardin JC, Solari A. Trypanosoma cruzi: variability of stocks from Colombia determined by molecular karyotype and minicircle southern blot analysis. Exp Parasitol. 2006;1 13:62-6.

65. Branche C, Ochaya S, Åslund L, Andersson B. Comparative karyotyping as a tool for genome structure analysis of Trypanosoma cruzi. Mol Biochem Parasitol. 2006;147:30-8.

66. Franzén O, Ochaya S, Sherwood E, Lewis MD, Llewellyn MS, Miles MA, et al. Shotgun sequencing analysis of Trypanosoma cruzi i Sylvio X10/1 and comparison with T. cruzi VI CL Brener. PLoS Negl Trop Dis. 2011;5:1-9.
67. El-Sayed NM. Comparative genomics of Trypanosomatid parasitic Protozoa. Science 2005;309:404-9. Available from: http://www.sciencemag.org/cgi/ doi/10.1126/science.1112181

68. Baptista RP, Reis-Cunha JL, DeBarry JD, Chiari E, Kissinger JC, Bartholomeu DC, et al. Assembly of highly repetitive genomes using short reads: the genome of discrete typing unit III Trypanosoma cruzi strain 231. Microb Genomics [Internet]. 2018; Available from: http://www.microbiologyresearch. org/content/journal/mgen/10.1099/mgen.0.000156.v1

69. Dujardin JC, Mannaert A, Durrant C, Cotton JA. Mosaic aneuploidy in Leishmania: the perspective of whole genome sequencing. Trends Parasitol. 2014;30:554-5 Available from: https://doi.org/10.1016/j.pt.2014.09.004.

70. Lachaud L, Bourgeois N, Kuk N, Morelle C, Crobu L, Merlin G, et al. Constitutive mosaic aneuploidy is a unique genetic feature widespread in the Leishmania genus. Microbes Infect. 2014;16:61-6.

71. Lima FM, Souza RT, Santori FR, Santos MF, Cortez DR, Barros RM, et al. Interclonal variations in the molecular karyotype of Trypanosoma cruzi: chromosome rearrangements in a single cell-derived clone of the $\mathrm{G}$ strain. PLoS One. 2013;8.

72. Mannaert A, Downing T, Imamura H, Dujardin JC. Adaptive mechanisms in pathogens: universal aneuploidy in Leishmania. Trends Parasitol. 2012; 28:370-6.

73. Selmecki A, Forche A, Berman J. Genomic plasticity of the human fungal pathogen Candida albicans. Eukaryot Cell. 2010;9:991-1008.

74. Hassold T, Hunt P. To err (meiotically) is human: the genesis of human aneuploidy. Nat Rev Genet [Internet] 2001;2:280-291. Available from: http:// www.ncbi.nlm.nih.gov/entrez/query.fcgi?cmd=Retrieve\&db=PubMed\&dopt= Citation\&list_uids $=11283700$

75. Stankiewicz P, Lupski JR. Structural variation in the human genome and its role in disease. Annu Rev Med [Internet] 2010;61:437-455. Available from: http://www.annualreviews.org/doi/10.1146/annurev-med-100708-204735

76. Lv L, Zhang T, Yi Q, Huang Y, Wang Z, Hou H, et al. Tetraploid cells from cytokinesis failure induce aneuploidy and spontaneous transformation of mouse ovarian surface epithelial cells. Cell Cycle. 2012;11:2864-75.

77. Doubre H, Césari D, Mairovitz A, Bénac C, Chantot-Bastaraud S, Dagnon K, et al. Multidrug resistance-associated protein (MRP1) is overexpressed in DNA aneuploid carcinomatous cells in non-small cell lung cancer (NSCLC). Int J Cancer. 2005;113:568-74.

78. Abbey D, Hickman M, Gresham D, Berman J. High-Resolution SNP/CGH Microarrays Reveal the Accumulation of Loss of Heterozygosity in Commonly Used Candida albicans Strains. G3 (Bethesda). [Internet]. 2011;1: 523-530. Available from: http://www.pubmedcentral.nih.gov/articlerender. fcgi?artid $=3276171 \&$ tool $=$ pmcentrez\&rendertype $=$ abstract

79. Leprohon P, Légaré D, Raymond F, Madore É, Hardiman G, Corbeil J, et al. Gene expression modulation is associated with gene amplification, supernumerary chromosomes and chromosome loss in antimony-resistant Leishmania infantum. Nucleic Acids Res. 2009;37:1387-99.

80. Weir W, Capewell P, Foth B, Clucas C, Pountain A, Steketee P, et al. Population genomics reveals the origin and asexual evolution of human infective trypanosomes, elife. 2016:5:1-14.

81. Berriman $\mathrm{M}$, Ghedin $\mathrm{E}$, Hertz-fowler $\mathrm{C}$. The genome of the African trypanosome, Trypanosoma brucei. Science. 2005;309(5733):416-22.

82. Ivens AC. The genome of the Kinetoplastid parasite, Leishmania major. Science [Internet] 2005;309:436-442. Available from: http://www. sciencemag.org/cgi/doi/10.1126/science.1112680\%5Cnpapers3:// publication/doi/10.1126/science.1112680

83. Clayton CE. Gene expression in Kinetoplastids. Curr Opin Microbiol. 2016;32: 46-51 Available from: https://doi.org/10.1016/j.mib.2016.04.018.

84. Günzl A, Bruderer T, Laufer G, Schimanski B, Tu LC, Chung HM, et al. RNA polymerase I transcribes procyclin genes and variant surface glycoprotein gene expression sites in Trypanosoma brucei. Eukaryot Cell. 2003;2:542-51.

85. Teixeira SM, de Paiva RMC, Kangussu-Marcolino MM, DaRocha WD. Trypanosomatid comparative genomics: contributions to the study of parasite biology and different parasitic diseases. Genet Mol Biol. 2012; 35:1-17.

86. Sheltzer JMJ, Blank HHM, Pfau SJS, Tange Y, George BM, Humpton TJ, et al. Aneuploidy drives genomic instability in yeast. Science [Internet]. 2011;333: 1026-30. Available from: http://www.sciencemag.org/content/333/6045/ 1026.abstract

87. Ubeda J-M, Légaré $\mathrm{D}$, Raymond $\mathrm{F}$, Ouameur AA, Boisvert $\mathrm{S}$, Rigault $\mathrm{P}$, et al. Modulation of gene expression in drug resistant Leishmania is associated 
with gene amplification, gene deletion and chromosome aneuploidy. Genome Biol [Internet]. 2008;9:R115. Available from: http://www. pubmedcentral.nih.gov/articlerender.fcgi?artid=2530873\&tool= pmcentrez\&rendertype $=$ abstract

88. Cardoso MS, Junqueira C, Trigueiro RC, Shams-eldin H, Previato O, Mendonc $\mathrm{L}$, et al. Identification and Functional Analysis of Trypanosoma cruzi Genes That Encode Proteins of the Glycosylphosphatidylinositol Biosynthetic Pathway. PLoS Negl Trop Dis. 2013;7.

89. Buscaglia CA, Campo VA, ACC F, Di Noia JM. Trypanosoma cruzi surface mucins: host-dependent coat diversity. Nat Rev Microbiol. 2006;4:229-36.

90. De Pablos LM, Osuna A. Multigene families in Trypanosoma cruzi and their role in infectivity. Infect Immun. 2012;80:2258-64.

91. Bolger AM, Lohse M, Usadel B. Trimmomatic: a flexible trimmer for Illumina sequence data. Bioinformatics. 2014;30:2114-20.

92. Aslett M, Aurrecoechea C, Berriman M, Brestelli J, Brunk BP, Carrington M, et al. TriTrypDB: a functional genomic resource for the Trypanosomatidae. Nucleic Acids Res. 2009;38:457-62.

93. Souto RP, Fernandes O, Macedo AM, Campbell DA, Zingales B. DNA markers define two major phylogenetic lineages of Trypanosoma cruzi. Mol Biochem Parasitol. 1996;83:141-52.

94. Burgos JM, Altcheh J, Bisio M, Duffy T, Valadares HMS, Seidenstein ME, et al. Direct molecular profiling of minicircle signatures and lineages of Trypanosoma cruzi bloodstream populations causing congenital Chagas disease. Int J Parasitol. 2007;37:1319-27.

95. Zerbino DR, Birney E. Velvet: algorithms for de novo short read assembly using de Bruijn graphs. Genome Res. 2008;18:821-9.

96. Zerbino DR. Technologies. Curr Protoc Bioinforma. [Internet]. 2011;1-13. Available from: http://doi.wiley.com/10.1002/0471250953.bi1105s31

97. Myers EW. A whole-genome assembly of drosophila. Science [Internet]. 2000;287:2196-204 Available from: http://www.sciencemag.org/cgi/doi/10. 1126/science.287.5461.2196.

98. Miller JR, Delcher AL, Koren S, Venter E, Walenz BP, Brownley A, et al. Aggressive assembly of pyrosequencing reads with mates. Bioinformatics. 2008;24:2818-24.

99. Li H, Durbin R. Fast and accurate short read alignment with burrowswheeler transform. Bioinformatics. 2009;25:1754-60.

100. Li H. Aligning sequence reads, clone sequences and assembly contigs with BWA-MEM. arXiv Prepr. arXiv [Internet]. 2013;0:3. Available from: http://arxiv. org/abs/1303.3997

101. Li H, Handsaker B, Wysoker A, Fennell T, Ruan J, Homer N, et al. The sequence alignment/map format and SAMtools. Bioinformatics. 2009;25: 2078-9.

102. Altschul SF, Gish W, Miller W, Myers EW, Lipman DJ. Basic local alignment search tool. J Mol Biol [Internet] 1990;215:403-410. Available from: http:// www.sciencedirect.com/science/article/pii/S0022283605803602

103. Darzentas N. Circoletto: visualizing sequence similarity with Circos. Bioinformatics. 2010;26:2620-1.

104. Zhang H, Meltzer P, Davis S. RCircos : an R package for Circos 2D track plots. BMC Bioinformatics [internet]. BMC Bioinformatics. 2013;14:1.

105. Kent WJ. BLAT — the BLAST -like alignment tool. Genome Res. 2002;12: 656-64.

106. Edgar RC. MUSCLE: multiple sequence alignment with high accuracy and high throughput. Nucleic Acids Res. 2004;32:1792-7.

107. Castresana J. Selection of Conserved Blocks from Multiple Alignments for Their Use in Phylogenetic Analysis. Mol Biol Evol. 2000;17:540-52.

108. Posada D. jModelTest: Phylogenetic model averaging. Mol Biol Evol. 2008;25: 1253-6.

109. Guindon S, Delsuc F, Dufayard JF, Gascuel O. Estimating maximum likelihood phylogenies with PhyML. Methods Mol Biol. 2009;537:113-37.

110. Huson DH, Richter DC, Rausch C, Dezulian T, Franz M, Rupp R. Dendroscope: An interactive viewer for large phylogenetic trees. BMC Bioinformatics [Internet]. 2007;8:460. Available from: http://bmcbioinformatics. biomedcentral.com/articles/10.1186/1471-2105-8-460

111. Quinlan AR, Hall IM. BEDTools: a flexible suite of utilities for comparing genomic features. Bioinformatics. 2010;26:841-2.

112. Suzuki R, Shimodaira H. Pvclust: an R package for assessing the uncertainty in hierarchical clustering. Bioinformatics. 2006;22:1540-2.

113. Shimodaira H. An Approximately Unbiased Test of Phylogenetic Tree Selection. Syst Biol. 2002;51:492-508.

\section{Ready to submit your research? Choose BMC and benefit from:}

- fast, convenient online submission

- thorough peer review by experienced researchers in your field

- rapid publication on acceptance

- support for research data, including large and complex data types

- gold Open Access which fosters wider collaboration and increased citations

- maximum visibility for your research: over $100 \mathrm{M}$ website views per year

At BMC, research is always in progress.

Learn more biomedcentral.com/submissions 